\title{
The Prestige of Somatic and Mental Disorders: A Survey Among Health Professionals and a Representative General Population Sample
}

\author{
Gunn Pettersen ${ }^{1}$, Reidun Olstad ${ }^{1}$ and Jan H. Rosenvinge ${ }^{*, 2}$ \\ ${ }^{1}$ University Hospital of North Norway; ${ }^{2}$ University of Tromsø, Department of Psychology, Norway
}

\begin{abstract}
Objective: The purpose of the paper is to study the ranking of disorders according to their perceived importance. Previous studies suggest that rankings according to the perceived or attributed "value" or importance create informal hierarchies of disorders on normative attitudes about symptoms, treatment and outcome. In this work we studied disorder ranking in the general population and among health professionals, and some possible explaining factors. Data source: 1,127 adults representative of the Norwegian National Population Register participated, of whom 220 representatives were of the broad range of health professionals. Study design: Respondents completed a survey questionnaire within a cross-sectional design. Principal findings: Somatic disorders were given the highest rank, but mental disorders were ranked higher than in previous studies. Modest effects were found for explaining variables. Conclusion: The general population rankings of disorders do not differ greatly from rankings made by health professionals. The impact of personalized variables was modest, indicating the need for future studies to explore the impact of more social and culture variables.
\end{abstract}

Keywords: Disorders, ranking, prestige.

\section{INTRODUCTION}

Should a patient with an acute heart condition be treated more promptly or rapidly than a patient with a mental disorder or a chronic lung obstructive disorder? Previous studies [1-4] show that medical doctors think so. These studies also indicate that such priorities reflect the degrees of "prestige" according to certain assumptions or psychological attributions towards patients and their various disorders, and not only strict medical judgments. In particular, a ranking according to priority and "importance" has been ascribed to assumptions about 1) etiology, i.e. whether the causes are somatic or mental in nature, transparent, unclear, complex or even unknown, or attributed to the patients way of life, 2) symptoms, i.e. whether the disorder is affecting vital organs such as the heart and the brain, and whether symptoms for instance, certain skin diseases, make the patients appear ugly or disgusting, and 3) treatment and outcome i.e. whether treatment is definitively curative or not, and whether the course of illness is acute or more long-standing.

Previous research then indicates that diagnoses are far from pure medical tools, enabling medical doctors to make rational treatment choices and predictions and subsequently communicate them to fellow professionals. Diagnoses are equally much social entities, conveying social meanings or connotations and attitudes about the social standing of disorders and the patients suffering from them. Such meanings may adhere to universal or common sense opinions as well as reflect a particular professional culture. Building on the concepts of stigma $[5,6]$ introduced a social typology of

*Address correspondence to this author at the University of Tromsø, Department of Psychology, Huginbakken 32, N-9037 Tromsø, Norway; E-mail: janr@psyk.uit.no disorders, suggesting an informal hierarchy or ranking according to non-medical and often tacit criteria of importance. Thus, as a stigma refers to negative attributions or connotations attached to certain disorders and the individuals who suffer from them, an attached stigma may not necessarily be verbalized, yet it may act as a powerful mechanism accounting for a low informal ranking. In the worst scenario from a personal and societal point of view, this ranking may be at variance with objective needs for professional treatment and care. Studying informal rankings or hierarchies of disorders are important and are proving even more essential as diagnoses are being applied for administrative and economic purposes that may influence the right to allowances as well as the right to treatment within fixed time limits [7].

Such an informal hierarchy may create problems. A low position in the hierarchy may delay patients from having the kind of treatment that would have been justified from their medical condition. Also, a high position may give patients with "important" or "interesting" disorders, a priority of treatment that does not need to be justified from their medical condition. A possible consequence of social connotations may then be unjust differences in the access or quality of treatment services, as well as in the priority given to research on the etiology and treatment of certain disorders [2]. A ranking of disorders does not, however, need not to be negative per se. In a world of limited economical resources allocated to the health care services, some priorities must be made. This raises the issue of legitimacy of rankings.

Provided the validity of assuming a disparity between a professional culture and the cultural context at large, one source of legitimacy is whether medical doctor's rankings match the rankings in the general public opinion, and exploring this is the overall purpose of the present study. A 
match would add to the legitimacy in terms of understanding and acceptance of priorities that medical doctors make, while a gross mismatch may certainly question the legitimacy and rationality of priorities in medical practice.

Non-medical influences on priorities may relate to professional and personalized biases, but these have not been empirically tested in previous studies $[1,2]$. Still the impact of professional biases has been speculated [1] in terms of rankings reflecting medical doctor's understanding of their professional role as instrumental in nature, leading in favor of disorders that need a lot of technical equipment for their treatment. If this may be true, one may expect different rankings in the general public or in samples comprising a broader spectrum of professionals, who may be inclined to define their professional roles as more expressional. Personal biases concern whether rankings are influenced by for instance health habits, perception of one's ability to control own health, personal acquaintance with serious disorders, a perceived risk of becoming affected by certain disorders, or attitudes about a relation between catching a disorder and the morality and personal responsibility of life styles. Suggesting that such personal biases are evenly distributed in the population, one may expect rankings to be sample type independent.

The aim of this cross-sectional survey study is to test differences in how somatic, mental, psychosomatic, and addictive disorders are ranked in the general public and among a broad range of health professionals as well as to test the influence of personalized biases.

\section{MATERIALS AND METHODOLOGY}

\section{Design, Subjects and Procedure}

A questionnaire was distributed to 1000 males and 1000 females $\geq 18$ years in the Northern part of Norway, representing the general population with respect to age and level of education. A professional polling agency ("Norwegian Gallup") was responsible for the sample selection and the data collection. To increase response rate, all subjects took part in a lottery for five rewards of $\$ 100$ each. In total, 1127 individuals returned the questionnaire. No gender difference in response rate was found (men $57 \%, \mathrm{~N}=572$, women 55 $\%, \mathrm{~N}=550)$. In the active sample $(\mathrm{N}=1122), 38 \%$ had received higher education (college or university degree), 40 $\%$ had completed high school, while $22 \%$ had a lower level of education. Moreover, $22 \%$ reported being health professionals. The mean age was 44.2 years (SD 11.0, range 19-87). Men were significantly older ((45.4 years, SD 11.2) than women $(42.9, \mathrm{SD} 10.6)(\mathrm{t}(1097)=3.94, \mathrm{p}<0.001)$, but the effect size of 0.23 (Hedge's g) was low.

\section{Instrument}

All respondents provided information about their age, gender, educational background, health habits (i.e. regular smoking and physical activity), and their personal experience with family members with severe acute or chronic disorder. Perceptions about personal control over health were measured by the "Health Locus of Control" scale [8], and in the present study, Chronbach's $\alpha$ for internal consistency showed acceptable values, i.e. 0.65 (external and random locus of control), and 0.73 (internal locus of control).

To measure specific attitudinal aspects of ranking, 16 statements measured specific attitudes towards health and disorders. Each statement was scaled 1-10 (maximal disagreement) to whether prestige and importance are related to the perceptions of disorders as being acute/chronic, having a clear/unclear etiology, effective/uncertain cure, and whether the etiology is attributed to patient's life style, gender or social status.

20 disorders were selected, covering the four dimensions "male/female dominated", "acute/chronic course", "known/ unknown etiology", and treatment as being "highly effective/ unclear or unknown". To combat response bias and response sets, the 20 disorders were listed in a random order according to these dimensions. One negative, and two positively formulated questions measured the rank based on economical priorities (i.e. "if you had become the minister of health, select three disorders from the above list as your first, second, and third priority for spending a) more money on treatment services, and b) less money on", and "if \$ 100 million were given to clinical research, please select three disorders as your first, second, and third priority").

To compute a total rank the first, second, and third priority were summed up for each of the three ranking variables (i.e. "would give more money to treatment services", "would spend less money on" and "would give more money to fund research") for each of the 20 disorders. Then, the sum score was calculated by adding the score for "would give more money to" and "would give more money for funding research", and subtracting the score for "would spend less money on". Based on this sum score, a total rank was given to each disorder as well as to the grouping of disorders as mental (i.e. depression, eating disorders, anxiety disorders, and schizophrenia), psychosomatic (asthma, ulcer, and fibromyalgia) or addictive disorders (alcoholism and drug addiction) or somatic (all residuals). Four or more in subgroup ranking differences was operationally defined as meaningful and interpretable.

\section{Statistical Analyses}

Attitudinal aspects of ranking of the 20 selected disorders were analyzed for health professionals versus non-health professionals, for gender, for the age groups $<29$ years, 3059 years, and $\geq 60$ years, for smokers versus non-smokers, and for those with and without a personal experience of serious disorders in their close family. Group differences were analyzed with t-tests and using p-values $<0.05$ or better, and effect sizes (Hedge's g) were conventionally defined as low $(<0.50)$; medium $(>0.51)$ and large $(>0.70)$. Factors, which influenced the ranking of disorders in the bivariate analyses, were used in stepwise linear regression analyses.

\section{RESULTS}

On the whole, typical acute and life threatening disorders (i.e. breast cancer, aids, cardiac infarction, brain tumor, and brain stroke) were ranked highest (1-5), while disorders like sciatica, ulcer, alcoholism, appendicitis, and ankle fracture 
were ranked lowest (16-20) (Table 1). Interestingly, mental disorders like anxiety -and mood disorders were ranked almost equal (7 and 8) to somatic disorders like ovarian (9) and testicular cancer (11). Overall health professionals and people from the general public ranked the 20 disorders in the same manner. Still the picture is somewhat complex. For instance, health professionals ranked cardiac infarction lower (7) than others (3), but not different from women (6). Also health professionals ranked lung cancer lower (10) than others (5), yet equal to that of women (10) and non-smokers (7).

\section{Impact of Biases on Ranking of Specific Disorders}

Some exceptions from the overall picture indicate a tendency towards a high ranking of disorders that could have been perceived as becoming potentially personally relevant. Thus, women tended to rank ovarian cancer and eating disorders higher (5 and 9) than men (10 and 13), while men ranked lung cancer, cardiac infarction and testicular cancer higher $(3,1$, and 8 , respectively) than women $(10,6$, and 13 , respectively). Similarly, regular smokers gave higher priority to lung cancer (3) compared to non-smokers (7) (Table 1). Overall, illness experiences yielded no differences, yet some age differences related to age related disorders were found. Thus, while breast cancer was given top rankings (1) among those between 18-59 years of age (Table 1), older participants gave this disorder a much lower ranking (8). This was also the case for AIDS that was given a substantial lower rank (17) than among those aged 18-29 (5) and 30-59 (2). Curiously enough, increasing age produced higher rankings of anxiety disorders with a notable difference between the 18-19 year olds (9) compared with those 60 years of age or more (5), while an opposite pattern was observed for mood disorders (Table 1).

\section{Impact of Biases on Groups of Disorders}

Health professionals gave higher priority than others to mental disorders (4.20 SD 1.63 versus $3.76 \mathrm{SD} 1.52, \mathrm{~F}(1)=$ $13.96, \mathrm{p}<0.0001$, Hedge's $\mathrm{g}=0.30$ ). Also, health professionals ranked addictive disorder higher $(2.87 \mathrm{SD}$

Table 1. Diseases Listed According to Sum Scores and Ranks for Subgroups, Using the Ranking in the Total Material for Reference. A Sum Score was Computed by Adding the Score for "Would Give More Money to Treatment Services" and "Would Give More Money for Funding Research", and Subtracting the Score for "Would Spend Less Money on". A Negative Sum Score Indicates that More Individuals Voted for Spending Less Money than those Giving Priority to the Disease

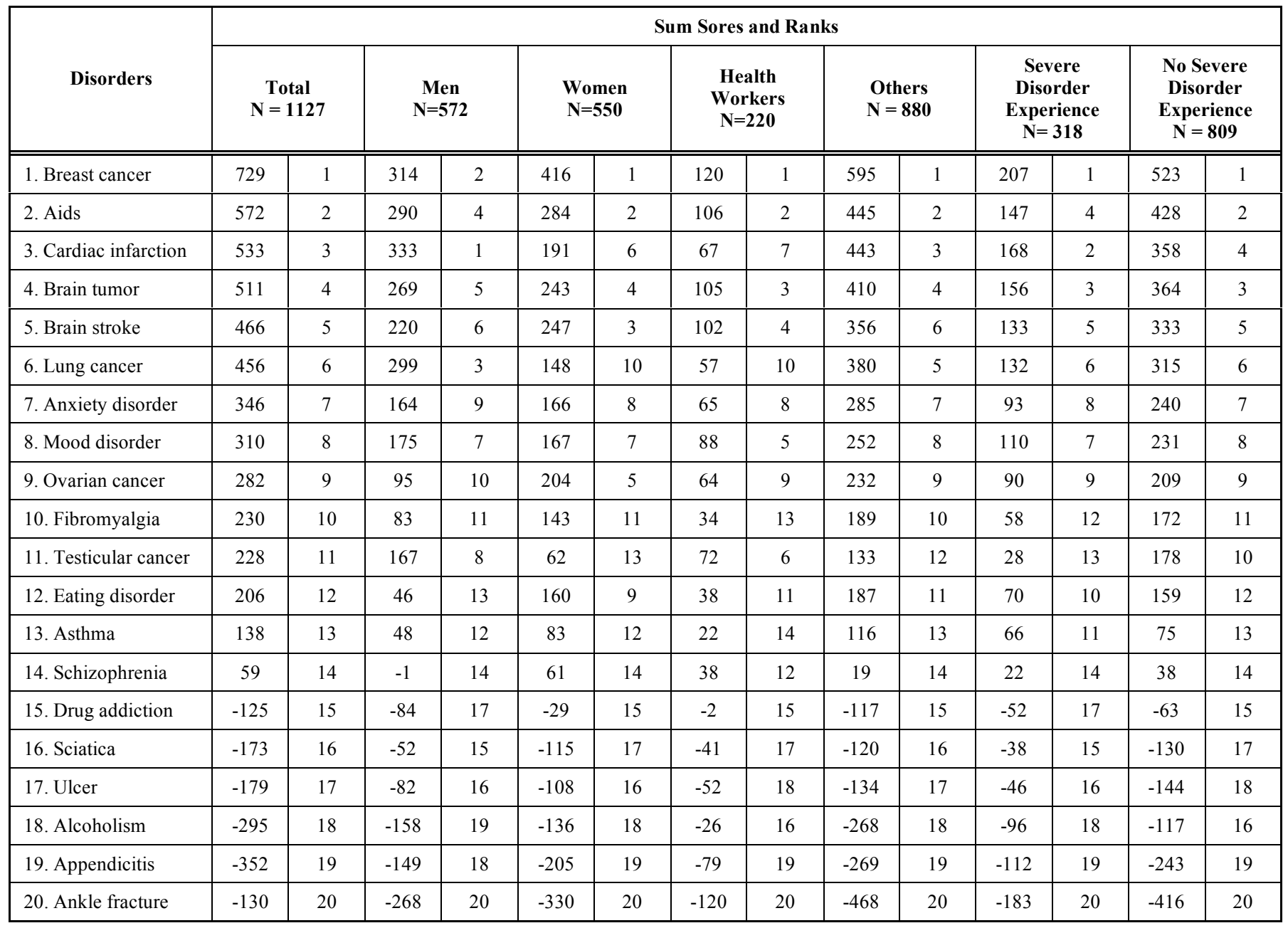


(Table 1) Contd.....

\begin{tabular}{|c|c|c|c|c|c|c|c|c|c|c|c|c|}
\hline \multirow{3}{*}{$\begin{array}{r}\text { Disorders } \\
\text { 1. Breast cancer }\end{array}$} & \multicolumn{12}{|c|}{ Sum Scores and Ranks } \\
\hline & \multicolumn{2}{|c|}{$\begin{array}{c}\text { Total } \\
\mathbf{N}=\mathbf{1 1 2 7}\end{array}$} & \multicolumn{2}{|c|}{$\begin{array}{c}\text { Regular smokers } \\
\mathbf{N}=\mathbf{3 9 3}\end{array}$} & \multicolumn{2}{|c|}{$\begin{array}{c}\text { Non-smokers } \\
\mathbf{N}=734\end{array}$} & \multicolumn{2}{|c|}{$\begin{array}{c}\text { Age 18-29 } y \\
N=103\end{array}$} & \multicolumn{2}{|c|}{$\begin{array}{c}\text { Age } 30-59 y \\
N=936\end{array}$} & \multicolumn{2}{|c|}{$\begin{array}{c}\text { Age } 60 y+ \\
N=61\end{array}$} \\
\hline & 729 & 1 & 244 & 2 & 486 & 1 & 40 & 1 & 599 & 1 & 13 & 8 \\
\hline 2. Aids & 572 & 2 & 257 & 1 & 318 & 4 & 21 & 5 & 455 & 2 & -1 & 17 \\
\hline 3. Cardiac infarction & 533 & 3 & 204 & 4 & 322 & 3 & 20 & 7 & 434 & 4 & 33 & 1 \\
\hline 4. Brain tumor & 511 & 4 & 192 & 5 & 328 & 2 & 15 & 11 & 439 & 3 & 5 & 15 \\
\hline 5. Brain stroke & 466 & 5 & 162 & 6 & 306 & 5 & 26 & 2 & 404 & 5 & 18 & 3 \\
\hline 6. Lung cancer & 456 & 6 & 206 & 3 & 241 & 7 & 21 & 6 & 367 & 6 & 30 & 2 \\
\hline 7. Anxiety disorder & 346 & 7 & 100 & 7 & 223 & 8 & 19 & 9 & 293 & 7 & 15 & 5 \\
\hline 8. Mood disorder & 310 & 8 & 91 & 9 & 264 & 6 & 19 & 8 & 292 & 8 & 9 & 12 \\
\hline 9. Ovarian cancer & 282 & 9 & 93 & 8 & 206 & 9 & 22 & 4 & 240 & 9 & 17 & 4 \\
\hline 10. Fibromyalgia & 230 & 10 & 76 & 12 & 153 & 11 & 23 & 3 & 188 & 10 & 10 & 10 \\
\hline 11. Testicular cancer & 228 & 11 & 76 & 11 & 154 & 10 & 15 & 12 & 172 & 12 & 14 & 6 \\
\hline 12. Eating disorder & 206 & 12 & 67 & 10 & 139 & 12 & 19 & 10 & 182 & 11 & 9 & 13 \\
\hline 13. Asthma & 138 & 13 & 30 & 13 & 111 & 13 & 8 & 13 & 122 & 13 & 13 & 9 \\
\hline 14. Schizophrenia & 59 & 14 & 18 & 14 & 42 & 14 & -4 & 16 & 56 & 14 & 14 & 7 \\
\hline 15. Drug addiction & -125 & 15 & -32 & 15 & -83 & 15 & 1 & 14 & -102 & 15 & 2 & 16 \\
\hline 16. Sciatica & -173 & 16 & -78 & 17 & -88 & 16 & -2 & 15 & -135 & 16 & -2 & 18 \\
\hline 17. Ulcer & -179 & 17 & -76 & 16 & -114 & 17 & -9 & 17 & -156 & 17 & 9 & 14 \\
\hline 18. Alcoholism & -295 & 18 & -96 & 18 & -200 & 18 & -17 & 18 & -241 & 18 & -10 & 20 \\
\hline 19. Appendicitis & -352 & 19 & -123 & 19 & -233 & 19 & -31 & 19 & -299 & 19 & 10 & 10 \\
\hline 20. Ankle fracture & -130 & 20 & -242 & 20 & -357 & 20 & -56 & 20 & -520 & 20 & -3 & 19 \\
\hline
\end{tabular}

1.16) than non-health professionals $2.56 \mathrm{SD} 1.10), \mathrm{F}(1)=$ 13.67, $\mathrm{p}<0.0001$, Hedge's $\mathrm{g}=0.30$ ). No differences were found for giving priority to psychosomatic disorders, while non-health professionals ranked somatic disorders higher (5.49 SD 2.09) than health professionals (4.89 SD 2.18), $(\mathrm{F}(1)=14.54, \mathrm{p}<0.0001$, Hedge's $\mathrm{g}=0.30)$. Professional background may however, be less important as higher priority to mental disorders was also related to being high (3.62 SD 1.26) versus medium (3.74 SD 1.54), and low (4.28 SD $1.62)$ educated $(\mathrm{F}(2)=36.10, \mathrm{p}<0.0001)$. Consistently, those with a low educational level favored giving priority to somatic disorders, (6.09 SD 1.79) than those with a medium (5.51 SD 2.10), and high level (4.80 SD 2.20; F (2) = 31.89, $\mathrm{p}<0.0001)$.

Within the effect size range $0.10-0.47$, women also gave higher priority to mental disorders (women 4.00 SD 1.52) than men (3.67 SD 1.58; F (1) = 13.18, p < 0.0001, Hedge's $\mathrm{g}=0.20)$, while men favored somatic disorders (5.66 SD 2.09) more than women (5.10 SD 2.13, F (1) $=20.61$, p < 0.0001 , Hedge's $g=0.20$ ). No gender or educational level differences in priority were found in the grouping variables "psychosomatic disorders", and "addictive disorders".

With respect to health habits, smokers and non-smokers did not give different priorities to the disorder groups, while being regularly physically active was related to giving higher priority to mental disorders compared to not being physically active (4.04 SD 1.62 versus 3.69 SD 1.50, F (1) = 14.05, p < 0.000 , Hedge's $g=0.20$ ). However, no statistically significant differences were found with respect to somatic, psychosomatic, and addictive disorders as well as for age or between those with and without a personal experience of serious illness in the family.

Four separate, stepwise regression analyses with "somatic disorders", "mental disorders", "psychosomatic disorders", and "addictive disorders" as the dependent variables yielded low beta values throughout. Thus, there was little predictive power for age, gender, educational level, being a health professional or not, health habits, and perceived health locus of control, and the regression models explained less than $10 \%$ of the variances (Tables $\mathbf{2 - 5}$ ).

\section{Impact of Biases on Attitudes About Status of Disorders}

There were no differences between health workers and the general population in judging high status of somatic disorders that are not as a result of a poor way of living, sport related injuries as well as disorders that are subject to much research. Moreover, no differences were found in judging fibromyalgia, geriatric disorders and eating disorders as having a lower status. 
Table 2. Stepwise Linear Regression Analysis for Variables Explaining Variance in the Ranking of Mental Disorders from 0-10 (Optimal)

\begin{tabular}{|c|l|c|c|}
\hline \multicolumn{2}{|l|}{ Step } & Standardized Beta & Adj R \\
\hline \hline 1. & Educational level & .24 & .06 \\
\hline 2. & Educational level & .23 & \\
\hline & Gender & .10 & .07 \\
\hline 3. & Educational level & .21 & \\
\hline & Gender & .10 & \\
\hline & Random locus of control & .10 & .07 \\
\hline 4. & Educational level & .20 & \\
\hline & Gender & .09 & \\
\hline & Random locus of control & .08 & \\
\hline & Extern locus of control & .06 & .08 \\
\hline
\end{tabular}

Table 3. Stepwise Linear Regression Analysis for Variables Explaining Variance in the Ranking of Somatic Disorders from 0-10 (Optimal)

\begin{tabular}{|c|l|c|c|}
\hline \multicolumn{2}{|l|}{ Step } & Standardized Beta & Adj R \\
\hline \hline 1. & Educational level & -.23 & .05 \\
\hline 2. & Educational level & -.21 & \\
\hline & Gender & -.13 & .07 \\
\hline 3. & Educational level & -.19 & \\
\hline & Gender & -.12 & \\
\hline & Random locus of control & -.12 & .08 \\
\hline
\end{tabular}

Table 4. Stepwise Linear Regression Analysis for Variables Explaining Variance in the Ranking of Psychosomatic Disorders from 0-10 (Optimal)

\begin{tabular}{|c|l|c|c|}
\hline \multicolumn{2}{|l|}{ Step } & Standardized Beta & Adj R $^{2}$ \\
\hline \hline 1. & Poor health habits & -.07 & .00 \\
\hline 2. & Poor health habits & -.21 & \\
\hline & Health professionals & -.13 & .00 \\
\hline 3. & Poor health habits & -.19 & \\
\hline & Health professionals & -.12 & \\
\hline & Gender & -.12 & .01 \\
\hline
\end{tabular}

Table 5. Stepwise Linear Regression Analysis for Variables Explaining Variance in the Ranking of Addictive Disorders from 0-10 (Optimal)

\begin{tabular}{|l|l|c|c|}
\hline \multicolumn{2}{|l|}{ Step } & Standardized Beta & Adj R $^{2}$ \\
\hline \hline 1. & Random locus of control & .14 & .02 \\
\hline 2. & Random locus of control & -.21 & \\
\hline & Health professionals & -.13 & .03 \\
\hline
\end{tabular}

Women agreed more (3.94 SD 3.10) than men (5.55 SD $3.12)$ to the statement that female disorders rank lower in prestige than male disorders, a significant difference $(\mathrm{p}<$ $0.0001)$ with a moderate $(0.51)$ effect size. Consistently, they disagreed more (7.63 SD 2.62) than men (6.73 SD 2.53; $\mathrm{p}<$ 0.0001 ) in viewing fibromyalgia as a high status disorder, and with an effect size (0.40) close to moderate (Table 6).

Other comparisons also reached statistical significance, but only with a low $(\leq 0.49)$ effect size. For instance compared to non-health workers, health professionals disagreed more to the statement that lung cancer has a high status than health care workers, even if the cause is smoking, and to statements rendering rare disorders and curable disorders a lower rank. Also, health professionals disagreed more to the statement that eating disorders and fibromyalgia have a high rank, and that sports related injuries have a low rank. A regression analysis showed a minor effect of gender, age, occupation (health workers or not), as well as a low and inconsistent impact on the health locus of control variables. Thus, the strongest effect was found for older respondents, who tended to view cardiac infarction as "the most important disorder in the health care services" (standardized $\beta=0.13, p$ $<0.0001$ ), but still the regression models explained less than $10 \%$ of the variances, ranging from $0.01-0.07$ (adjusted $\mathrm{R}^{2}$ ).

\section{DISCUSSION}

The study aim was to investigate the ranking of somatic, mental, psychosomatic, and addictive disorders in the general population and among health professionals, as well as the impact of possible explanatory variables.

We found that a high rank was given to acute somatic disorders with a known etiology and where high-technology treatment is expected to be effective. This is in accord with previous studies among medical doctors $[1,2]$. However, mental disorders were given a substantially high rank among a broad range of health professionals. This finding is strikingly different from two previous studies $[1,2]$ among medical doctors conducted in the same cultural domain within a 17 -years time span. Here, medical doctors rated mental disorders lowest. At least in part, the difference may be biased by the listing of disorders. In previous research [2], the vast majority of disorders were somatic in nature, and the high priorities and rankings of such disorders would result in a dramatic drop in the probability of high rankings of mental disorders. In the current study, the distribution of disorders intended to reduce this bias, thus giving an "equal chance" of being selected for a high ranking. Other reasons for the difference between the current and previous findings relate to a possible professional medical subculture disfavoring mental disorders. This hypothesis is supported by the fact that in the current study, high ranking of mental disorders was related to being higher educated, which is also present in a sample of medical doctors. Our findings may indicate a more open minded attitude towards mental disorders in the society as well as among nom-medical health professions, and that a possible disfavoring of mental health problems among medical doctors lacks legitimacy.

This "upgrading" of mental disorders in terms of ranking of priority and the recognition of these disorders as important is certainly echoed by health statistics of prevalence and 
Table 6. Means and SD for 16 Fixed Statements about Disorder Rankings for the Total Material and by Subgroups. $1=$ maximal Agreement, 10 = Maximal Disagreement. Means are Arranged in Ascending Order with the Total Sample as Reference

\begin{tabular}{|c|c|c|c|c|c|}
\hline & $\begin{array}{c}\text { Total } \\
\mathbf{N}=1127 \\
\mathbf{M}(\mathbf{S D})\end{array}$ & $\begin{array}{c}\text { Men } \\
\mathrm{N}=\mathbf{5 7 2} \\
\mathrm{M} \text { (SD) }\end{array}$ & $\begin{array}{l}\text { Women } \\
N=\mathbf{5 5 0} \\
M(\text { SD) }\end{array}$ & $\begin{array}{c}\text { Health workers } \\
\mathbf{N}=\mathbf{2 2 0} \\
\mathrm{M} \text { (SD) }\end{array}$ & $\begin{array}{l}\text { Others } \\
N=880 \\
M(\text { SD) }\end{array}$ \\
\hline $\begin{array}{l}\text { Somatic disorders have a higher status than mental } \\
\text { disorders }\end{array}$ & $3.18(2.66)$ & $3.33(2.65)$ & $3.00(2.62)^{*} \times$ & $3.13(2.78)$ & $3.16(2.58)$ \\
\hline $\begin{array}{l}\text { Disorders caused by a poor way of life are less } \\
\text { prestigious }\end{array}$ & $3.42(2.56)$ & $3.44(2.61)$ & $3.38(2.45)$ & $3.45(2.58)$ & $3.40(2.51)$ \\
\hline $\begin{array}{l}\text { Disorders that are subject to much research gives them } \\
\text { high prestige }\end{array}$ & $3.94(2.93)$ & $4.02(2.91)$ & $3.83(2.91)$ & $4.05(3.08)$ & $3.88(2.86)$ \\
\hline $\begin{array}{l}\text { Cardiac infarction is the most important disease in the } \\
\text { health services }\end{array}$ & $4.19(2.57)$ & $4.29(2.54)$ & $4.07(2.57)$ & $4.20(2.70)$ & $4.20(2.51)$ \\
\hline $\begin{array}{l}\text { Easily cured mental disorders have a higher status than } \\
\text { those that are difficult to treat }\end{array}$ & $4.26(2.49)$ & $4.26(2.47)$ & $4.24(2.48)$ & $4.11(2.69)$ & $4.26(2.40)$ \\
\hline $\begin{array}{l}\text { Using technological equipment in treatment is highly } \\
\text { prestigious }\end{array}$ & $4.30(2.92)$ & $4.44(2.89)$ & $4.13(2.90)$ & $4.23(3.19)$ & $4.30(2.82)$ \\
\hline $\begin{array}{l}\text { Lung cancer has a high status even if the cause is } \\
\text { regular smoking }\end{array}$ & $4.42(2.72)$ & $4.40(2.72)$ & $4.43(2.70)$ & $5.00(2.91)$ & $4.25(2.61)^{* * * 0}$ \\
\hline $\begin{array}{l}\text { The patient's social status impact how disorders are } \\
\text { given priority }\end{array}$ & $4.74(3.13)$ & $4.72(3.13)$ & $4.74(3.10)$ & $4.83(3.08)$ & $4.683 .11)$ \\
\hline $\begin{array}{l}\text { The prestige of disorders depends on whether the cause } \\
\text { is clearly defined }\end{array}$ & $4.76(2.47)$ & $4.73(2.45)$ & $4.76(2.46)$ & $4.94(2.72)$ & $4.69(2.37)$ \\
\hline $\begin{array}{l}\text { "Female" disorders rank lower in prestige than "male" } \\
\text { disorders }\end{array}$ & $4.78(3.23)$ & $5.55(3.12)$ & $3.94(3.10) * * * \#$ & $4.51(3.26)$ & $4.81(3.20)$ \\
\hline Rare disorders rank low in prestige & $5.58(2.88)$ & $5.61(2.93)$ & $5.52(2.81)$ & $6.01(2.81)$ & $5.43(2.87)^{* * \alpha}$ \\
\hline $\begin{array}{l}\text { Disorders that can be cured are less prestigious than } \\
\text { chronic disorders }\end{array}$ & $5.68(3.02)$ & $5.57(2.98)$ & $5.78(3.03)$ & $6.46(3.12)$ & $5.47(2.95)^{* * * 0}$ \\
\hline Eating disorders have a high status & $6.60(2.51)$ & $6.48(2.48)$ & $6.71(2.53)$ & $6.95(2.46)$ & $6.50(2.49) * * \alpha$ \\
\hline Sports related illnesses and injuries rank low in prestige & $6.8982 .73)$ & $6.81(2.78)$ & $6.95(2.68)$ & $7.11(2.64)$ & $6.82(2.74)$ \\
\hline Fibromyalgia has a high status & $7.18(2.62)$ & $6.73(2.53)$ & $7.63(2.62)^{* * * a}$ & $7.55(2.65)$ & $7.09(2.57)^{* * a}$ \\
\hline $\begin{array}{l}\text { Disorders among elderly have a higher status than those } \\
\text { affecting younger }\end{array}$ & 7.75 (2.49) & $7.68(2.46)$ & $7.81(2.51)$ & $7.86(2.53)$ & $7.71(2.48)$ \\
\hline
\end{tabular}

\begin{tabular}{|c|c|c|c|c|}
\hline & $\begin{array}{l}\text { Severe disorder } \\
\text { experience } \\
\mathbf{N}=\mathbf{3 1 8} \\
\mathbf{M}(\mathrm{SD})\end{array}$ & $\begin{array}{c}\text { Others } \\
\mathbf{N}=\mathbf{8 0 9} \\
\mathbf{M} \text { (SD) }\end{array}$ & $\begin{array}{l}\text { Regular } \\
\text { smokers } \\
\mathrm{N}=\mathbf{3 9 3} \\
\mathrm{M}(\mathrm{SD})\end{array}$ & $\begin{array}{c}\text { Others } \\
\mathbf{N}=734 \\
\mathbf{M} \text { (SD) }\end{array}$ \\
\hline Somatic disorders have a higher status than mental disorders & $3.00(2.57)$ & $3.24(2.69)$ & $3.04(2.82)$ & $3.25(2.74)$ \\
\hline Disorders caused by a poor way of life are less prestigious & $3.54(2.64)$ & $3.25(2.47)$ & $3.65(2.66)$ & $3.30(2.55) * 0$ \\
\hline Disorders that are subject to much research gives them high prestige & $3.73(2.86)$ & $4.04(3.05)$ & $3.98(2.98)$ & $3.92(2.90)$ \\
\hline Cardiac infarction is the most important disease in the health services & $4.13(2.59)$ & $4.22(2.63)$ & $4.41(2.66)$ & $4.07(2.51)^{*} \mathrm{~d}$ \\
\hline Easily cured mental disorders have a higher status than those that are difficult to treat & $3.97(2.36)$ & $4.36(2.54) * 0$ & $4.44(2.66)$ & $4.16(2.39)$ \\
\hline Using technological equipment in treatment is highly prestigious & $4.25(2.97)$ & $4.28(2.90)$ & $4.26(2.87)$ & $4.32(2.94)$ \\
\hline Lung cancer has a high status even if the cause is regular smoking & $4.21(2.71)$ & $4.33(2.62)$ & $4.50(2.86)$ & $4.37(2.65)$ \\
\hline The patient's social status impact how disorders are given priority & $4.71(3.08)$ & $4.86(3.18)$ & $4.88(3.29)$ & $4.67(3.04)$ \\
\hline The prestige of disorders depends on whether the cause is clearly defined & $4.59(2.51)$ & $4.79(2.44)$ & $4.67(2.51)$ & $4.80(2.55)$ \\
\hline "Female" disorders rank lower in prestige than "male" disorders & $4.61(3.23)$ & $4.81(3.22)$ & $4.79(3.23)$ & $4.77(3.23)$ \\
\hline Rare disorders rank low in prestige & $5.55(2.82)$ & $6.94(2.70)$ & $5.76(2.94)$ & $6.48(2.85)$ \\
\hline Disorders that can be cured are less prestigious than chronic disorders & $5.54(3.09)$ & $5.73(2.98)$ & $5.79(3.02)$ & $5.62(3.01)$ \\
\hline Eating disorders have a high status & $6.49(2.42)$ & $6.51(2.49)$ & $6.58(2.52)$ & $6.61(2.51)$ \\
\hline Sports related disorders and injuries rank low in prestige & $6.70(2.82)$ & $6.94(2.70)$ & $6.97(2.81)$ & $6.90(2.69)$ \\
\hline Fibromyalgia has a high status & $7.04(2.74)$ & $7.13(2.55)$ & $7.18(2.68)$ & $7.18(2.58)$ \\
\hline Disorders among elderly have a higher status than those affecting younger & $7.63(2.55)$ & $7.65(2.49)$ & $7.65(2.67)$ & $7.80(2.39)$ \\
\hline
\end{tabular}

$* \mathrm{p}<0.04 ; * * \mathrm{p}<0.01 ; * * * \mathrm{p}>0.000 ; \mathrm{d}=$ low effect size $(<0.5)$; $\#=$ medium effect size $(\geq 0.5)$ (Hedge's $g$ ). 
burdens. Mental disorders are common in the United States and internationally, amounting to one in four adults above 18 years of age - or about 60 million Americans suffering from a diagnosable mental disorder in a given year. Moreover, mental disorders are the leading cause of disability in the U.S. and Canada for ages 15-44, and the Global Burden of Disease study [9] shows that mental disorders severely affect health and productivity in the U.S. and worldwide. Here, mental disorders, including suicide, account for over $15 \%$ of the burden of disease in established market economies, such as in the U.S. This is more than the disease burden caused by all cancer disorders [10].

The overall impact of variables relevant to test the personalized bias hypothesis was generally low. Yet some aspects deserve to be mentioned. As for the gender perspective, women tended to agree more than men that disorders that are attributed to the female gender are ranked lower in the health services. Broken down in specific disorders yielded an inconsistent picture as no gender differences were found in the ranking of breast cancer and the attitudinal statements related to disorders generally attributed to the female gender, like eating disorders and fibromyalgia. On the other hand, larger and gender specific differences were found with respect to ovarian cancer and testicular cancer in terms of ranking.

As for disorders related to life style, the general trend was a support to the idea that disorders attributed to a poor or negative life style are less prestigious (Tables 2 and $\mathbf{3}$ ). Hence, health care professionals tended not to favor a notion that lung cancer caused by smoking is important. Still, smokers tended to disagree more to the statement that disorders related to a poor life style are less prestigious (Table 2), and smokers also gave lung cancer a higher status than nonsmokers (Table 1). This finding may lend support to a notion that a favorable ranking or attitude is related to a personalized bias, yet AIDS was top ranked throughout (except for those $>60$ years of age) despite the fact that AIDS is not expected to be especially personally relevant in this general population sample.

There is an almost universal downhill trend in response rates of general population surveys. Also in the catchment area for the current study, the response rates have decreased steadily from about $63 \%$ in 1987 to $34 \%$ in $2002[11,12]$. At least in our catchment area, a low response rate may not imply a selection bias in large samples, as non-response appears to occur at random [11-13], thus not seriously introducing systematical errors deflating the external validity. Assuming that this applies to the current study, the fact that a polling agency selected a representative sample and that the response rate is well above that of mainstream surveys, lends weak arguments for judging the response rate as a serious limitation in our study.

Future research may take several pathways to explore issues of practical and theoretical importance. First, there is a need to replicate in order to resolve inconsistencies of findings. Secondly, there is a need to explore ranking differences across professional subcultures, and to scrutinize rankings and in fact, the "social value" of disorders in the social context where such rankings actually are made. Such contexts may range from clinical evaluations, the making of waiting lists for patient groups as well as calculating social benefits and health insurances. Third, there is a need to study ranking of disorders where the selection of disorders may vary systematically. To control for the effect of selecting and listing per se is important as any kind of listing may convey a social message. Finally, the present study focused on personal factors' impact on ranking, yet such factors did not explain much. Thus, a change of focus to a broader, sociological perspective and theories of social constructionism may help us to further understand how "personal" ranking results are affected by the norms, values and attitudes in the community the mutual interaction between individuals and the society. In this perspective, at least in retrospect it may border pseudoempiricism to explore differences in ranking within a culture as social constructivism may predict that subgroup differences would be just marginal. A preferred strategy for future research within this theoretical frame may be to study rankings cross-culturally.

\section{CONCLUSION}

The general population rankings of disorders do not differ greatly from rankings made by health professionals. The impact of personalized variables was modest, indicating the need for future studies to explore the impact of more social and culture variables.

\section{REFERENCES}

[1] Album D. The prestige of diseases and medical specialties. J Nord Med Assoc 1991; 111: 2127-33.

[2] Album D, Westin S. Do diseases have a prestige hierarchy? A survey among physicians and medical students. Soc Sci Med 2008; 66: 181-8.

[3] Schwartzbaum AM, McGrath JH, Rothman RA. The perception of prestige differences among medical subspecialties. Soc Sci Med 1973; 7: 365-71.

[4] Shortell SM. Occupational prestige dfferences within the medical and allied health professions. Soc Sci Med 1974; 8: 1-9.

[5] Goffman E. Stigma. Englewood Cliffs, NJ: Prentice-Hall 1963.

[6] Freidson E. Professions of medicine: a study of the sociology of applied Knowledge. New York: Dodd Mead \& Co 1970.

[7] Hart TJ. The political economy of health care: a clinical perspective. Bristol: The Policy Press 2006.

[8] Levenson H. Multidimensional locus of control in psychiatric patients. J Consult Clin Psychol 1973; 41: 397-404.

[9] WHO, The burden of diseases. [Accessed 0407 08] Available from: www.who.int/ topics/global_burden_of_diseases/en

[10] NIMH The Global Burden of Disease study. [Accessed 0407 08] Available from: www.nimh.gov/health statistics/index/shtml

[11] Hellevik O. Some ethical problems in research using quantitative methods. Oslo: The National Committees for Research Ethics Norway $1999 ; 12 ; 37-48$.

[12] Traeen B, Holmen K, Stigum H. Extradyadic sexual relationships in Norway. Arch Sex Behav 2007; 36: 55-65.

[13] Søgaard AJ, Selmer R, Bjertness E, Thelle D. The Oslo health survey: the impact of self-selection in a large, population-based survey. Int J Equity Health 2004; 3: 1-12. 